\title{
Primary Thyroid Tuberculosis
}

\author{
Sunita Sanehi • Chandrashekhar Dravid • Neena Chaudhary • A. K. Rai
}

\begin{abstract}
In India, it is estimated that more than $40 \%$ of the adults are infected with tuberculosis bacilli and every year 2 million people develop tuberculosis and nearly 500,000 die from it ${ }^{1}$. But, tuberculosis of the thyroid gland occurs only rarely. Since extra-pulmonary tuberculosis is now seen relatively more frequently, the existence of this condition should be recognized when goitres are being treated. We present a case of right thyroid nodule associated with low grade fever and weight loss. No signs and symptoms of hypothyroidism or hyperthyroidism were present. Patient did not have any other tuberculosis focus. Routine investigation and chest X-ray were normal. Sputum for Acid Fast Bacilli was negative. Fine Needle Aspiration Cytology and Contrast Enhanced Computed Tomography led to a diagnosis of Primary Thyroid Tuberculosis in a euthyroid patient. Patient responded well to Anti tubercular Drug Therapy along with repeated aspiration.
\end{abstract}

Key words Tuberculosis $\cdot$ Thyroid Gland $\cdot$ Euthyroid

Sunita Sanehi $(\square)$

Senior Research Fellow

Pocket - A-1, MIG Flat No. - 94,

Sector - 7, Rohini,

Delhi - 110085, India.

E-mail : dr.sunita@hotmail.com

Tel: 011 - 26192038

Chandrashekhar Dravid

Senior Resident

Neena Chaudhary

Specialist

A. K. Rai

Consultant \& Head of Department

Department of ENT, Safdarjung Hospital,

New Delhi - 110029, India.

\section{Introduction}

Tuberculosis of the thyroid gland is extremely uncommon with very few cases reported so far. Thyroid tuberculosis is usually diagnosed by physicians in $0.1-1 \%$ of cases. But during autopsy this disease is found much more often (2 to $7 \%$ of cases). This is explained by difficulties of diagnosing the disease according to its symptoms and by insufficient knowledge of physicians on the subject of differential diagnosis of the thyroid tuberculosis ${ }^{2}$. Symptom - free thyroid involvement may occur as part of generalized miliary spread or, more rarely, diffuse or localized swelling of the gland may dominate the clinical picture ${ }^{3}$. We report a case of Primary Thyroid Tuberculosis in a euthyroid patient presenting as a thyroid nodule.

\section{Gase Report}

A 45-year-old female patient presented with a slow, progressively increasing painless swelling over right side of midline of neck. This swelling had been present for at least 5 months. She complained of low grade fever and loss of about $4 \mathrm{~kg}$ of body weight over the same time. There were no symptoms of stridor, dysphagia or hoarseness. Moreover, there were no signs and symptoms of thyroid dysfunction. There was no past or family history of tuberculosis or thyroid disease.

Examination revealed an average built female with low grade pyrexia. There was a swelling $6 \times 5 \mathrm{~cm}$ in size, present on the anterior aspect of the neck more towards the right side. The margins of the swelling were well defined and it had a smooth surface with firm consistency. Rest of the gland was normal. It was mobile on swallowing. No cervical lymph nodes were palpable and the overlying skin was normal. It was not adherent to the skin or adjacent structures. Trachea was slightly pushed to the left side. There were no bruits or thrills. No lymphadenopathy was palpable. Systemic examination was normal. She was clinically euthyroid.

A diagnosis of solitary nodule of right lobe of thyroid gland 
Fig. 1 Radiogram of neck and upper chest AP view, showing a well defined soft tissue mass on the right side of the neck compressing and shifting the trachea to the opposite side.

Fig. 2 Contrast Enhanced CT Scan Neck, axial view showing a peripheral enhancing low density abscess in the right thyroid lobe.
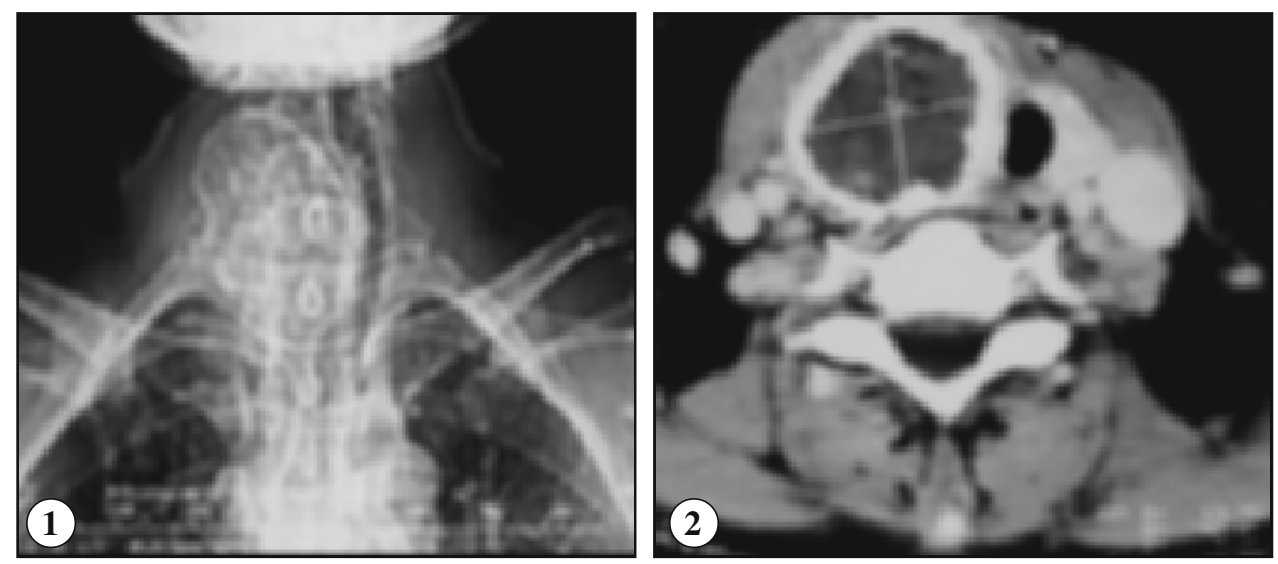

was made in a euthyroid patient. Investigations revealed a normal haemogram except a raised ESR of $40 \mathrm{~mm}$ in the first hour. The liver and kidney function tests were within normal limits. $\mathrm{T}_{3} \mathrm{~T}_{4}$ and TSH levels were normal. An assay for Purified Protein Derivative (PPD) was positive $(18 \mathrm{~mm}$ x $18 \mathrm{~mm}$ ). Analysis of sputum for AFB was negative. Lung fields on both sides were clear on X-ray chest. FNAC from the swelling revealed that the mass consisted of granuloma with caseous necrosis along with langhan's giant cells and epithelioid cells. Culture for AFB was positive. The chest X-ray neck AP view (Fig. 1) revealed a swelling on the right side of the neck with well defined margins compressing and shifting the trachea to the left side.

Contrast Enhanced Computed Tomography (Fig. 2) scan revealed a peripherally enhancing low density swelling involving the right lobe of the thyroid gland. On the basis of histopathological and radiological investigation, we established the diagnosis of Primary Thyroid Tuberculosis.

Patient was started on standard anti tubercular drugs (W.H.O. - category 1) i.e, Rifampicin, Ethambutol, Isoniazid and Pyrazinamide for initial phase of 2 months followed by continuation phase with Rifampicin and Isoniazid for next 4 months. Repeated aspiration of the necrotic material from the mass was also done. Patient responded well to the treatment and there was gradual resolution of the thyroid swelling. She was symptom free and the swelling disappeared completely after 6 months.

\section{Discussion}

In the $19^{\text {th }}$ century, it was authoritatively suggested that tuberculosis never involved the thyroid gland and that there was some antagonistic action between goitre and tuberculosis. The relative immunity of the thyroid gland has been confirmed but has not been explained ${ }^{3}$. Primary involvement of the thyroid gland is difficult to explain. The latent focus of infection perhaps is of great importance in the development of such type of extra-pulmonary tuberculosis. This is in contrast to the thyroid involvement associated with pulmonary or extra-pulmonary tuberculosis, where spread of the disease occurs by haematogenous, or lymphogenous and likely to occur when infection is progressive ${ }^{4}$.

Lebert (1862) reported the first case of tuberculosis of thyroid involvement in a patient with disseminated miliary tuberculosis ${ }^{3}$. Chiari (1878) described seven cases of microscopic involvement of thyroid in 100 autopsies of patients who had died from disseminated tuberculosis ${ }^{3}$. Bruns in 1893 described the first case of tuberculosis thyroiditis diagnosed in a middle aged woman with a rapidly enlarging goitre, who had cervical lymphadenopathy but no evidence of pulmonary tuberculosis ${ }^{3}$. The first report of successful drainage of tuberculosis thyroid abscess was by Schwartz in $1894^{3}$. Five cases of thyroid tuberculosis were described by Coller and Huggins $(1926)^{5}$ in a series of 1200 cases of thyroid disease operated on between 1920 and 1926; all of these, however, showed miliary tuberculosis occurring in preexisting goitres. Enlargement of the cervical lymph nodes in both anterior and posterior chains were found to be present in a moderate number of cases in the literature (Coller and Huggins $)^{5}$. Rankin and Graham $(1932)^{6}$ studied a large series of 20758 partial thyroidectomy specimens from the Mayo Clinic and diagnosed tuberculosis in only 21 cases, an incident of $0.1 \%$. Our patient had gradually enlarging goitre without any cervical lymphadenopathy. There was no evidence of pulmonary tuberculosis or involvement of any other organ in the body. Only E.S.R. (40 mm) was raised and PPD was strongly positive.

Tuberculosis may involve the thyroid gland mainly in two forms. The more common of these is miliary spread to the thyroid as part of generalized dissemination but this form has never been shown to give rise to clinical thyroid disease (Rankin \& Graham, 1932) ${ }^{6}$. Occasionally, miliary spread may occur in pre-existing thyroid enlargement (Coller and Huggins, 1926) ${ }^{5}$. Less commonly focal or caseous tuberculosis of the thyroid may occur, presenting as a localized swelling mimicking carcinoma (Crompton and Cameron, 1969) ${ }^{7}$.

Disorders of thyroid function have seldom been described in association with tubercular thyroiditis. Mosiman (1917) recorded nine cases in which seven were said to be thyrotoxic on clinical grounds, although no biochemical confirmation 
was possible. Indeed it has been suggested that thyrotoxicosis affords some protection against infection by mycobacterium tuberculosis (Joll, 1932) ${ }^{9}$. Hypothyroidism as a result of progressive thyroid destruction may be expected to occur but in the few cases in which indices of thyroid function has been measured biochemically normal values have been obtained ${ }^{3}$. In our case, there were no signs and symptoms of hyper or hypothyroidism. $\mathrm{T}_{3}, \mathrm{~T}_{4}$ and TSH levels were normal.

Definite criteria for the disease were not established and ranged from rather loose descriptions to the rigid requirements of Seed ${ }^{10}$. He required that the diagnosis of the tubercular abscess of the thyroid to be made only - 1) when Acid Fast Bacilli are found, 2) in a necrotic or an abscessed thyroid gland, and 3) with a definite etiologic focus in the body outside the thyroid. Using these criteria, he was unable to find gross or microscopic evidence of tuberculosis. Jaffe (1930) ${ }^{11}$ reported 21 cases in 300 thyroid glands surgically removed in which nodular structures were found, composed of epithelioid cells and giant cells strongly resembling tuberculosis. Pina Sacca ${ }^{12}$ attempted to distinguish between the two types on histologic grounds. Schelling ${ }^{13}$ introduced the diagnosis of "Giant Cell Variant" and Holtz ${ }^{14}$ presented such a case.

Histological and bacteriological confirmation is adequate to make the diagnosis and fulfillment of the third criterion is not essential. It is now stated that Acid Fast Bacilli is not always present on microscopic examination, therefore multiple coalesced and caseated tubercles and epithelioid cell granuloma, are considered positive for tubercular affection of the gland. Mosimann ${ }^{8}$, Plummer and Broders ${ }^{15}$ and most of the subsequent investigators have continued to accept the histological evidence as the diagnostic criterion. The importance of FNAC was further affirmed by Mondal and Potra ${ }^{16}$ in 1995 . Our patient presented with a solitary nodule and was subjected to FNAC. It revealed the characteristic features of epithelioid cell granuloma, langhan's giant cells and also demonstrated Acid Fast Bacilli.

Ultrasonographic and computed tomographic findings can help in this matter as well: heterogenous hypoechoic mass is seen on ultrasonogram and peripheral enhancing low density abscess with regional lymphadenopathy is demonstrated on CT $\operatorname{scan}^{2}$. Contrast Enhanced Computed Tomography Scan of our patient revealed a peripherally enhancing low density abscess involving the right lobe of the thyroid gland.

Treatment of thyroid tuberculosis is complex : administration of anti-tubercular drugs is combined with surgical removal of the affected parts of thyroid gland or surgical drainage. Repeated puncture drainage and antitubercular drugs are the least invasive mode of treatment. Lately treatment with the antitubercular drugs has been recognized as the preferred method. In case of tuberculous abscess in the thyroid, drainage is sufficient, surgery is required rarely ${ }^{2}$. In our case the patient was put on antitubercular therapy along with repeated aspirations. A positive response was seen with a complete disappearance of the swelling in 6 months.

\section{Summary}

Tuberculosis should be included in the differential diagnosis of the thyroid swelling. The disease can manifest in various ways. There are no specific symptoms characteristics only to thyroid tuberculosis. The main method of establishing diagnosis is Fine Needle Aspiration Cytology with subsequent bacterioscopical, bacteriological or biological investigations. Still for verification of diagnosis ultrasonography, even computerized tomography and the newest serological diagnostic methods of tuberculosis may be necessary. It is particularly vital to distinguish thyroid tuberculosis from thyroid cancer, in an attempt to avoid unnecessary thyroid surgery. It can be effectively treated by repeated aspiration along with anti tubercular drugs.

\section{References}

1. Group at Risk: Report on the Tuberculosis Epidemic WHO TB/96. World Health Organization, Geneva, 1996

2 Simkus Andrius (2004): Medicina 40: 3; 201 - 203

3. Barnes Peter and Weatherstone Robert (1979): Tuberculosis of the Thyroid : Two Case Reports. Br J Dis Chest 73: 187 191

4. Hazard J.B. (1955): Thyroiditis : A review. Am J Clin Path 25: 189

5. Coller F.A., Huggins C.B. (1926): Tuberculosis of the thyroid gland. Annals of Surgery 84: 804

6. Rankin F.W. and Graham A.S. (1932): Tuberculosis of the thyroid gland. Ann. Surg. 96: 625

7. Compton G.K. and Cameron S.J. (1969): Tuberculosis of the thyroid gland mimicking carcinoma. Tubercle 50: 61

8. Mosiman R.E. (1917): Tuberculosis of the thyroid. Surgery Gynec. Obstet. 24: 680

9. Joll C.A. (1932): Diseases of the Thyroid Gland. London: Heinemann

10. Seed L. (1939): In: Goldberg's Clinical Tuberculosis, $2^{\text {nd }}$ Edition, Philadelphia, F.A. Davis Co.

11. Jaffe R.H. (1930): Tubercle like structures in human goitre. Arch. Surg. 21: 717

12. Pina Sacca F. (1949): Histologic distinction between subacute parenchymatous thyroiditis and tuberculosis. Minerva med. 1: 334

13. Schelling J.A. (1945): Struma lymphomatosa, struma fibrosa and thyroiditis. Surg. Gynec. Obst. 81: 533

14. Holtz R. (1947): Struma fibrosa - giant cell variant. Ohio State M. J. 43: 11530

15. Plummer W.A. and Broders A.C. (1920): Tuberculosis of the Thyroid. Minnesota Med., 3: 279 - 283

16. Mondal A, Potra D.K. (1995): Efficiency of fine needle aspiration in the diagnosis of tuberculosis of thyroid gland, 18 cases. J Laryngol Otol 109(1): 36 - 38 